SU-ITP-05/01

DFPD-05/TH/02

hep-th/0501081

\title{
Dirac Action on M5 and M2 Branes with Bulk Fluxes
}

\author{
Renata Kallosh ${ }^{1}$ and Dmitri Sorokin ${ }^{2}$ \\ ${ }^{1}$ Institute for Theoretical Physics, Department of Physics \\ Stanford University, Stanford, CA 94305 \\ kallosh@stanford.edu \\ ${ }^{2}$ INFN Sezione di Padova \& Dipartimento di Fisica "Galileo Galilei", \\ Università degli Studi di Padova, 35131, Padova, Italy \\ dmitri.sorokin@pd.infn.it
}

\begin{abstract}
We derive an explicit form of the quadratic-in-fermions Dirac action on the M5 brane for an arbitrary on-shell background of 11D supergravity with non-vanishing fluxes and in presence of a chiral 2-form on M5. This action may be used to generalize the conditions for which the non-perturbative superpotential can be generated in $\mathrm{M} /$ string theory. We also derive the Dirac action with bulk fluxes on the M2 brane.
\end{abstract}




\section{Introduction}

Our purpose is to derive the quadratic action for fermions on the M5 brane in a background of 11D supergravity with generic fluxes. Although the action of M5 in supergravity background 1 as well as the equations of motion for all fields on M5 surface are known [2, 3, 4], an explicit dependence on 4-form fields of 11D supergravity in the Dirac action on M5 has not been given yet.

The motivation for this work is two-fold. First of all to find an explicit form of a Dirac operator in presence of generic fluxes for the fundamental brane objects may have its own merit, especially if the answer is reasonably simple and allows to understand implications for the physics of the branes in such backgrounds. For the M2 and Dp branes such studies have already been undertaken in the literature. For instance, the coupling of D3 brane fermions to type IIB supergravity fluxes was studied in [5]. And in [6. 7] Dp brane actions in generic supergravity backgrounds were derived in the quadratic approximation for fermions (in the Green-Schwarz form without gauge fixing worldvolume reparametrization and kappa-symmetry) from a corresponding M2 brane action [8] using T-duality ${ }^{1}$. In this paper we present the results for the M5 brane and also for the M2 brane both in the Green-Schwarz and purely worldvolume form. This will allow one to investigate, in particular, various matrix models in backgrounds which are not necessarily of an $A d S \times S$-type but more general ones.

Secondly, significant part of the motivation for this work was the recent interest in nonperturbative corrections to M/string theory. It has been argued by Witten in [13] a while ago that the $S O(2)$ symmetry, which is a subgroup of the structure group $S O(5)$ may be the exact symmetry of the M5 brane action, no background fluxes were considered at that time. This was a basis for establishing a very powerful theorem about the conditions when non-perturbative superpotentials can be generated in $\mathrm{M} /$ string theory.

The analysis is based on algebraic geometry and leads to the statement that the compactification four-fold must admit divisors of arithmetic genus one, $\chi_{D} \equiv \sum(-1)^{n} h_{n}=1$. This indicates that in type IIB compactifications there can be non-vanishing corrections to the superpotential coming from Euclidean D3 branes. In the presence of such instantons, there is a correction to the superpotential which at large volume yields a term $W_{\text {inst }}=T\left(z_{i}\right) \exp (2 \pi i \rho)$ where $T\left(z_{i}\right)$ is a one-loop determinant, and the leading exponential dependence comes from the action of a Euclidean D3 brane wrapping a four-cycle in the compactified manifold. The non-perturbative corrections to the superpotential are absent, according to [13, when $\chi_{D} \neq 1$ (in absence of fluxes).

The presence of such non-perturbative corrections to the superpotential plays a crucial role in the stabilization of the volume modulus, as was shown in the simplest KKLT model with one Kähler modulus [14] and in a general class of models with many Kähler moduli in [15]. In particular, in all models of Denef, Douglas and Florea in [15] the choice of compactification manifolds was always satisfying the restriction that $\chi_{D}=1$ and a significant effort was made to find them.

An analysis of some of these issues was performed in [16] where the role of the 4-flux in the generation of instanton corrections has been discussed. In particular it was shown that the total flux through the divisor must vanish.

It was suggested by Gorlich, Kachru, Tripathy and Trivedi in [17] and argued for particular examples of compactification that in the presence of fixed fluxes the $U(1)$

\footnotetext{
${ }^{1}$ Other aspects of Dirac operators on branes have been considered e.g. in [9]-[12].
} 
symmetry of the fermionic action of the M5 brane might be broken. This in turn leads to a possibility of generating non-perturbative superpotentials in models with divisors on a four-fold of an arithmetic genus $\chi_{D} \geq 1$.

There are two possible points of view on $U(1)$ symmetry in presence of fluxes. These correspond to two well known aspects of symmetries of the background functional in field theory [18]. One is related to the so-called "quantum gauge transformations" and the second one is related to the "background gauge transformations" accompanied by the corresponding transformations of the quantum fields (variables of integration). In our case when the $U(1)$ symmetry acts as "quantum gauge transformations" only the fermions on the M5 brane are transformed but background fluxes are kept fixed. In presence of fixed background fluxes the corresponding $U(1)$ symmetry on the M5 brane may be broken. On the other hand, the $U(1)$ symmetry acting as the "background gauge transformations" in our case means that the fluxes, if they are vectors or tensors in the structure group, transform simultaneously with the action of $U(1)$ on fermions. In this sense the classical Dirac action on the brane has an unbroken $U(1)$ symmetry in presence of the background fluxes.

In the setting used in [13] we find it useful to consider fluxes transforming together with fermions under the $U(1)$-symmetry so that the classical invariance of the M5 brane action takes place. This symmetry of the classical theory may be broken by one-loop anomalies. However, with account of the inflow from the bulk these anomalies are expected to be canceled [13] since the $U(1)$ is just a part of diffeomorphisms and the theory is expected to be exactly invariant.

This all development is suggestive that the exact dependence of the Dirac operator on M5 with fluxes may help to understand the situation in a completely general setting since with account of bulk fluxes the algebraic properties of the M5 brane Dirac operator will change. To find the corresponding changes we need to find an explicit contribution of bulk fluxes to the Dirac operator on the M5 brane. We may proceed in two ways.

1. First we may look for the fermion action in a target space covariant Green-Schwarz form where the corresponding anti-commuting fields are worldvolume scalars and target space spinors of $\operatorname{Spin}(1,10)$. For the reader who just wants to see the effect of the flux $F_{a b c d}$ on M5 and M2 fermions, here is the simplified form (without tensor field couplings on the M5 brane) of the Dirac equation for the 16-component 'kappa-projected' spinor in the background with fluxes. Note that in the approximation without tensor fields Dirac equations have the same form for M5 and M2

$$
\Gamma^{a}\left(\nabla_{a}+T_{a} \underline{a b c d} F_{\underline{a b c d}}\right) \theta_{-}=0 .
$$

Here $T_{a} \underline{a b c d}$ stands for a product of $\gamma$-matrices (the detailed notation is introduced below).

2. We may look for the action for the world-surface fermions transforming in $\operatorname{Spin}(1.5) \times$ $\operatorname{Spin}(5)$. This form is more useful for the future studies of the instanton effects from the Euclidean M5 brane wrapped on a six-cycle of a Calabi-Yau 4-fold [13]. We will need eventually to perform an analytic continuation to Euclidean space with spinors in $\operatorname{Spin}(6) \times \operatorname{Spin}(5)$. Here again is the simplified form of the Dirac equation for the chiral spinor on the M5 brane

$$
\left[\tilde{\gamma}^{a} \nabla_{a}+\frac{1}{24}\left(\gamma^{i j k} \tilde{\gamma}^{a} F_{a i j k}-\gamma^{i} \tilde{\gamma}^{a b c} F_{a b c i}\right) \theta\right]_{q}^{\alpha}=0,
$$

see notation and details in the paper. An analogous equation is also given for the M2 brane below. 
The derivation of the explicit form of the quadratic-in-fermions Dirac action on the M5 brane for an arbitrary on-shell background of 11D supergravity with non-vanishing fluxes and tensor fields on the M5 brane and on the M2 brane and of the corresponding equations of motion is explained below.

Since we are looking for a quadratic part of the action in a non-trivial background we may simply look at fermionic equations of motion which in a general form can be obtained either using superembedding techniques [2] or directly from the M5-brane action [1, 3]. We will follow notation of the review paper [4].

In Sec. 2 we derive the Green-Schwarz type fermionic equations of the M5 brane in a flux background for space-time spinors being world-volume scalars. In Sec. 3 we derive Dirac equations for the world-volume spinors in a flux background. In both cases $\kappa$ symmetry is effectively gauge-fixed, so that the relevant fermion is 16-component. In Sec. 4 we present the Dirac action on M5 in a flux background with examples of how $U(1)$ symmetry acts on the fluxes. Dirac equations and the corresponding Lagrangian with bulk fluxes on the M2 brane are given in Sec. 5. In conclusion some final comments are made. Appendix contains some useful technical details on gamma-matrices and Lorentz spinor harmonics.

\section{Green-Schwarz type fermionic equations of the M5 brane}

We start with the M5 brane fermionic equation in the Green-Schwarz form

$$
\frac{1}{2} m^{b a} E_{\bar{a}}^{\underline{\beta}}\left[E_{b}^{\underline{a}} \Gamma_{\underline{a}}(1-\bar{\Gamma})\right]_{\underline{\beta} \underline{\alpha}}=0 .
$$

In eq. (3)

$$
E_{\bar{a}}^{\underline{\beta}}=e_{a}^{m}(\xi) \partial_{m} Z^{\underline{M}}(\xi) E_{\underline{\underline{M}}}^{\underline{\beta}}(Z), \quad E_{\bar{a}}^{\underline{a}}=e_{a}^{m}(\xi) \partial_{m} Z^{\underline{M}}(\xi) E_{\underline{\underline{M}}}^{\underline{a}}(Z)
$$

are the pullbacks on the M5 brane worldvolume, parametrized by the coordinates $\xi^{m}$ ( $m=0,1, \cdots, 5)$, of the $D=11$ supergravity supervielbeins

$$
E^{\underline{A}}(Z)=d Z^{\underline{M}} E_{\underline{\underline{M}}}^{\underline{A}}=\left(E^{\underline{\alpha}}, E^{\underline{a}}\right)
$$

where $Z \underline{M}=\left(x \underline{\underline{m}}, \theta^{\underline{\mu}}\right)(\underline{m}=0,1, \cdots, 10 ; \underline{\mu}=1, \cdots, 32)$ are local coordinates of curved $D=11$ superspace $^{2}$.

$e_{a}^{m}(\xi)$ is the inverse vielbein on the M5 brane worldvolume associated with the induced worldvolume metric $g_{m n}=\partial_{m} Z \underline{M} E_{\underline{M}}^{\underline{a}} \partial_{n} Z \underline{\underline{N}} E_{\underline{\underline{N}}}^{\underline{b}} \eta_{\underline{a b}}$. As so, $E_{a} \underline{a}$ satisfy the orthogonality condition

$$
E_{a} \underline{a} E_{b} \underline{b} \eta_{\underline{a b}}=\eta_{a b}
$$

where $\eta_{\underline{a b}}$ and $\eta_{a b}$ are respectively $D=11$ and $d=6$ Minkowski metric.

\footnotetext{
${ }^{2}$ In our notation the underlined indices correspond to $D=11$ target superspace and not underlined ones correspond to the M5 brane worldvolume. The indices from the beginning of the Latin and Greek alphabet are vector and spinor tangent (super)space indices, while the indices from the middle of the Latin and Greek alphabet are that of local curved coordinates.
} 
Finally, the matrix $m^{a b}=m^{b a}=\eta^{a b}-2 h^{a}{ }_{c d} h^{b c d}$ describes the interaction of the fermionic (and bosonic) worldvolume fields with the self-dual world--volume tensor $h_{a b c}=$ $\frac{1}{3 !} \epsilon_{a b c d e f} h^{d e f}$. The tensor $h_{a b c}$ is related via the nonlinear equation

$$
4\left(m^{-1}\right)_{a}^{d} h_{d b c}=H_{a b c}
$$

to the field strength $H^{(3)}=\frac{1}{3 !} e^{c} e^{b} e^{a} H_{a b c}=d b^{(2)}(\xi)-A^{(3)}$ of the M5 brane chiral 2form gauge field $b^{(2)}(\xi)$ extended with the pull back of the 3 -form gauge potential $A^{(3)}$ of $D=11$ supergravity whose field strength

$$
F^{(4)}=d A^{(3)}=\frac{i}{2} E^{\underline{a}} E^{\underline{b}} \bar{E} \Gamma_{\underline{b a}} E+\frac{1}{4 !} E^{\underline{a}_{4}} \ldots E^{\underline{a}_{1}} F_{\underline{a}_{1} \cdots \underline{a}_{4}}
$$

generates the background fluxes. $\Gamma_{\underline{\alpha}}^{\underline{\alpha}}$ are $D=11$ gamma-matrices in the Majorana representation and $\bar{\Gamma}$ is their antisymmetrized product

$$
\bar{\Gamma}=\frac{1}{6 !} \epsilon^{a_{1} \cdots a_{6}} \Gamma_{a_{1} \cdots a_{6}}+\frac{1}{3} h^{a b c} \Gamma_{a b c}, \quad \Gamma_{a}=E \frac{a}{a} \Gamma_{\underline{a}},
$$

such that $\bar{\Gamma}^{2}=1$.

Equation (3) is invariant under the $\kappa$-symmetry transformations

$$
\delta_{\kappa} Z^{\underline{M}} E_{\underline{\underline{M}}}^{\underline{\alpha}}=\frac{1}{2}(1+\bar{\Gamma})_{\underline{\alpha}}^{\underline{\alpha}} \kappa^{\underline{\beta}}, \quad \delta_{\kappa} Z^{\underline{M}} E_{\underline{\underline{M}}}^{\underline{a}}=0 .
$$

Kappa-symmetry allows one to eliminate half of the M5 brane fermionic degrees of freedom. To see this one may notice that the right hand side of (13) is annihilated by the $\kappa$-symmetry projector $\frac{1}{2}(1+\bar{\Gamma})^{\underline{\alpha}}{ }_{\underline{\beta}}$.

To extract the explicit depen $\bar{d}$ ence on fluxes in the Dirac equation on the M5 brane in the linear approximation for the fermions we have to evaluate the ingredients in this equation in the corresponding approximation. We look at

$$
E_{\bar{m}}^{\frac{\beta}{m}}=\partial_{m} Z^{\underline{M}} E_{\underline{\underline{M}}}^{\frac{\beta}{\underline{M}}}=\partial_{m} Z^{\underline{\mu}} E_{\underline{\underline{\mu}}}^{\underline{\beta}}+\partial_{m} Z^{\underline{m}} E_{\underline{\underline{m}}}^{\frac{\beta}{}} .
$$

We now define

$$
\theta \underline{\beta} \equiv Z \underline{\underline{\mu}} E_{\underline{\mu}}^{\underline{\beta}}
$$

and rewrite eq. (111) in the following form

$$
E_{\bar{m}}^{\underline{\beta}}=\partial_{m} \theta^{\underline{\beta}}-Z \underline{\mu} \partial_{m} E_{\underline{\underline{\mu}}}^{\underline{\beta}}+\partial_{m} Z^{\underline{m}} E_{\underline{\underline{m}}}^{\frac{\beta}{1}}
$$

The first term is already at the linear level in $\theta$ and provides the free Dirac equation in the flat $D=11$ background. Without coupling to the bosonic fields and to the tensor field on the brane we would find, for $m^{b a}=\delta^{b a}, e_{b}^{m}=\delta_{b}^{m}$ and $E \frac{a}{n}=\delta_{n}^{a}$,

$$
E_{\bar{a}}^{\underline{\beta}}\left[\Gamma^{a}(1-\bar{\Gamma})\right]_{\underline{\beta} \underline{\alpha}}=\partial_{a} \theta \underline{\beta}\left[\Gamma^{a}(1-\bar{\Gamma})\right]_{\underline{\beta} \underline{\alpha}}=0,
$$

If we introduce the chiral spinor $\theta_{-}=\frac{1}{2}(1-\bar{\Gamma}) \theta$, which is manifestly invariant under $\kappa$-symmetry transformations (10), we find a simple Dirac equation

$$
\Gamma^{a} \partial_{a} \theta_{-}=0
$$


This equation in turn easily transfers into a free Dirac equation for a chiral spinor on the M5 brane. However, here we would like to take care of corrections due to background geometry and in particular bulk fluxes. We therefore should evaluate the remaining terms in eq. (13).

In the Wess-Zumino gauge the term $\partial_{m} E_{\underline{\mu}}^{\underline{\beta}}$, that has to be evaluated at the zero order in $\theta$ since it is multiplied by $\theta^{\mu}$, vanishes. The third term in eq. (13) provides us with the information we are looking for. The vector-spinor vielbein $E_{\underline{\underline{m}}}^{\frac{\beta}{}}$ in (11|32) superspace starts with gravitino $\psi \underline{\underline{m}}(x)$, which we shall not take into account below restricting ourselves to pure bosonic $D=11$ supergravity backgrounds. The term linear in $\theta$ was calculated long time ago in [19]: this term is proportional to the rhs of the gravitino supersymmetry transformation excluding the term with the space-time derivative acting on the supersymmetry parameter ${ }^{3}$. In notation of [4]

$$
E \underline{\underline{\underline{\beta}}}=\psi \underline{\underline{\underline{m}}}+\left(\Omega \underline{\underline{\underline{m} \alpha}}+T_{\underline{\underline{m} \alpha}}^{\underline{\beta}}\right) \theta^{\underline{\alpha}}+\ldots
$$

where the term linear in $\theta$ includes a $\operatorname{Spin}(1,10)$ connection

$$
\Omega_{\underline{\underline{m} \alpha}}^{\underline{\beta}}=\left(\frac{1}{4} \Omega \underline{\underline{a b}} \Gamma_{\underline{a b}}\right)^{\underline{\beta}} \underline{\underline{\alpha}}
$$

and the flux-dependent superspace torsion term

$$
T_{\underline{\underline{m} \alpha}}^{\beta}=\left(T_{\underline{\underline{m}}}^{\underline{a b c d}} F_{\underline{a b c d}}\right){ }_{\underline{\alpha}}^{\beta} .
$$

Here

$$
T \frac{a b c d e}{288}\left(\Gamma \frac{1}{a b c d e}-8 \eta^{\underline{a}[\underline{b}} \Gamma \underline{c d e]}\right) .
$$

Thus we get

$$
m^{b a} e_{b}^{m} e_{a}^{n}\left(\partial_{m} \theta^{\underline{\beta}}+\left(\Omega_{\underline{m}}^{\underline{\beta}}+T_{\bar{m} \underline{\alpha}}^{\underline{\beta}}\right) \theta^{\underline{\alpha}}\right)\left[E_{\bar{n}}^{\underline{a}} \Gamma_{\underline{a}}(1-\bar{\Gamma})\right]_{\underline{\beta \gamma}}=0
$$

In the absence of $11 \mathrm{D}$ fluxes $F_{\underline{a b c d}}$ and of the M5 brane chiral gauge field, and in the approximation in which the fermionic equation is linear in $\theta$, it is simply the Dirac equation with a metric compatible spin connection and an $S O(5)$ gauge group connection encoded in the covariant derivative $\nabla_{a}$

$$
\Gamma^{a} \nabla_{a} \theta_{-}=0
$$

When fluxes are present we find that

$$
m^{a b} \Gamma_{b}\left(\nabla_{a}+T_{a} \underline{a b c d} F_{\underline{a b c d}}\right) \theta_{-}=0 .
$$

Equation (22) is of a Green-Schwarz type in the sense that the fermionic field and the fluxes carry the target superspace vector and spinor indices. To reduce it to an equation which describes the dynamics of the M5 brane fermionic modes in the effective $6 d$ worldvolume field theory one can, for example, impose on the worldvolume scalars the physical

\footnotetext{
${ }^{3}$ The $\theta^{2}$ term was given in [10], the $\theta^{3}$ term was derived in [8] and the expression for $E_{\underline{\underline{m}}}^{\frac{\beta}{2}}$ up to the 5 th order in $\theta$ was calculated in [20] using a compact expression for the Wess-Zumino gauge, analogous to the one proposed for $D=4$ supergravity in [21].
} 
(static) gauge, which fixes worldvolume reparametrization invariance, and eliminate half of the fermionic modes by gauge fixing $\kappa$-symmetry. Such a gauge fixing breaks $D=11$ local $\operatorname{Spin}(1,10)$ symmetry down to its subgroup $\operatorname{Spin}(1,5) \times \operatorname{Spin}(5)$, where $\operatorname{Spin}(1,5)$ is the $6 d$ worldvolume 'Lorentz' symmetry and $\operatorname{Spin}(5) \sim U S p(4) \sim S O(5)$ is the internal R-symmetry of the effective chiral $(2,0) d=6$ supersymmetric worldvolume field theory. This method was used in [9] where the free action for the tensor multiplets on the worldvolume of M5 brane was derived.

Alternatively, one can get the same worldvolume fermion equation in a simpler way, without breaking $D=11$ Lorentz invariance, by singling out the irreducible $\kappa$-invariant part of $\theta^{\underline{\alpha}}$ using the method of Lorentz harmonics which is part of the superembedding approach (see [24, 4]) for a review and references). In the next section we shall use the latter method to derive the purely worldvolume fermionic equation with fluxes.

\section{Dirac equation on M5 surface}

Here we start with another form of the fermionic equation

$$
\tilde{\gamma}_{b}^{\alpha \beta} m^{b a} E_{\bar{a}}^{\underline{\beta}} v_{\underline{\beta}, \beta q}=0,
$$

which is related to eq. (3) by a certain transformation [2, 4.

In eq. (23) $\tilde{\gamma}_{b}^{\alpha \beta}$ and $\gamma_{\alpha \beta}^{a}$ are antisymmetric $d=6 \operatorname{Spin}(1,5) \gamma$-matrices having the properties described in eqs. (41) - (42) of the Appendix, and $v_{\beta, \beta q}(\xi)$ are half of the components of the $\operatorname{Spin}(1,10)$ matrix (called Lorentz spinor harmonics)

$$
v_{\underline{\alpha}}^{\underline{\beta}}=\left(v_{\underline{\alpha}}^{\alpha p}, v_{\underline{\alpha}, \beta q}\right), \quad C \underline{\alpha} \underline{\gamma} v_{\underline{\alpha}}^{\underline{\beta}} v_{\underline{\underline{\gamma}}}^{\underline{\delta}}=C \underline{\beta} \underline{\delta}=\left(\begin{array}{cc}
0 & \delta_{\beta}^{\alpha} \delta_{q}^{p} \\
-\delta_{\gamma}^{\delta} \delta_{s}^{r} & 0
\end{array}\right) .
$$

In (24) the $\operatorname{Spin}(1,10)$ index $\underline{\beta}$ is split into the two pairs ${ }^{\alpha p}$ and ${ }_{\beta q}$ of indices of $\operatorname{Spin}(1,5) \times$ $\operatorname{Spin}(5)$ which is the symmetry of the M5 brane worldvolume theory. The corresponding realization of the $D=11 \Gamma$-matrices is given in the Appendix, eqs. (38)-(40). Note that the upper and lower $\operatorname{Spin}(1,5)$ indices ${ }^{\alpha}$ and ${ }_{\beta}$ correspond to inequivalent chiral spinor representations of $\operatorname{Spin}(1,5)$, and there is no a $6 d$ charge conjugation matrix which would raise and lower these indices.

The Lorentz harmonics (24) are auxiliary worldvolume fields. They are related to the pullback $E \frac{A}{a}(Z(\xi))$ of the $D=11$ supervielbein (5) by the equations (44)-(45) of the Appendix. In particular,

$$
E_{a} \underline{\alpha} v_{\underline{\alpha}}^{\alpha p}=0 .
$$

We can use the spinor harmonics (24) to convert the target space spinor field (12) into a pair of chiral and anti-chiral worldvolume spinors

$$
\theta^{\underline{\beta}} \underline{v}_{\beta} \beta q \equiv \theta_{\beta q}, \quad \theta^{\underline{\beta}} \underline{\underline{\beta}}^{\alpha p} \equiv \theta^{\alpha p} .
$$

Because of $\kappa$-invariance (which is reflected in the orthogonality condition (25)) the antichiral spinor field $\theta^{\alpha p}$ does not appear in the fermionic equation (23). In other words, one can use local $\kappa$-symmetry transformations to put $\theta^{\alpha p}$ to zero.

Then (23) takes the form similar to (22) but with $d=6$ worldvolume matrix $\tilde{\gamma}_{b}$ instead of pulled back $D=11$ matrix $\Gamma_{b}$

$$
\left[\tilde{\gamma}_{b} m^{b a}\left(\nabla_{a}+T_{a} \underline{a b c d} F_{\underline{a b c d}}\right) \theta\right]_{q}^{\alpha}=0 .
$$


Here the covariant derivative is the derivative with account of metric compatible spin connection for $\operatorname{Spin}(1,5) \times \operatorname{Spin}(5)$ structure group (see 4 for details).

Using the relations (38)-(45) of the Appendix we can rewrite (27) in a purely worldvolume form

$$
\tilde{\gamma}_{b}^{\alpha \beta} m^{b a} \nabla_{a} \theta_{\beta q}+\frac{1}{24}\left[\left(\gamma^{i j k} \tilde{\gamma}^{b}\left(2 \delta_{b}^{a}-m_{b}^{a}\right) F_{a i j k}+\gamma^{i} \tilde{\gamma}^{b c d}\left(2 \delta_{b}^{a}-3 m_{b}^{a}\right) F_{a c d i}\right) \theta\right]_{q}^{\alpha}=0,
$$

where the indices $i, j, k=1,2,3,4,5$ correspond to the target space directions transversal to the M5 brane.

If we ignore the 3 -form $h$ contribution, we find that in our approximation eq. (28) reduces to

$$
\tilde{\gamma}^{a \alpha \beta} \nabla_{a} \theta_{\beta q}+\frac{1}{24}\left[\left(\gamma^{i j k} \tilde{\gamma}^{a} F_{a i j k}-\gamma^{i} \tilde{\gamma}^{a b c} F_{a b c i}\right) \theta\right]_{q}^{\alpha}=0
$$

\section{Dirac action and examples of flux transforming under $U(1)$ symmetry}

The fermion Lagrangian which produces the equations (28) is

$$
L_{f}^{M 5}=\frac{1}{2} \theta\left[\tilde{\gamma}_{b} m^{b a} \nabla_{a}+\frac{1}{24}\left(\gamma^{i j k} \tilde{\gamma}^{d}\left(2 \delta_{d}^{a}-m_{d}^{a}\right) F_{a i j k}+\gamma^{i} \tilde{\gamma}^{b c d}\left(2 \delta_{d}^{a}-3 m_{d}^{a}\right) F_{a b c i}\right)\right] \theta .
$$

Let us note that via $m^{a b}$, which contains the self-dual field $h_{a b c}$ defined in (7), the M5 brane fermions couple directly, though non-minimally, to the pull back of the 3-form flux potential $A_{a b c}$. In the presence of the worldvolume flux $h_{a b c}$ both the self-dual and antiself-dual worldvolume parts of the flux $F_{a b c i}$ appear in the fermion Lagrangian, while if we neglect the contribution of $h_{a b c}$ (so that $m_{b}{ }^{a}=\delta_{b}{ }^{a}$ ), the flux $F_{a b c i}$ should be anti-self-dual on the M5 worldvolume, since $\tilde{\gamma}^{a b c}$ is self-dual (see eq. (43) of the Appendix).

The action in presence of fluxes is invariant under the $S O(5)$ structure group transformations with the flux $F_{a i j k}$ transforming as a $3 \mathrm{~d}$ rank antisymmetric tensor and the $F_{a b c i}$ transforming as an $S O(5)$ vector.

We may split the $S O(5)$ index into those of $S O(3)$ and $S O(2)$, namely $i=\mu, \nu, \lambda, I, J$. This corresponds to splitting 5 directions normal to the brane into $\mathbf{R}^{3}$ for the 3 directions $i=\mu, \nu, \lambda$ and the remaining two directions $i=I, J$ will correspond to $S O(2) \sim U(1)$. We will be interested in the situation that $F_{a i j k}$ has only $F_{a \mu \nu \lambda}$ components and $F_{a b c i}$ has only $F_{a b c I}$ components. This would correspond to the 4 -fold compactification of M-theory with 8 coordinates $a=1, \ldots, 6, I=1,2$ to a 3 -dimensional space with 3 coordinates $\mu, \nu, \lambda$.

The action is invariant under $S O(3) \times S O(2)$ symmetry with the flux $F_{a b c I}$ transforming as a vector under $S O(2)$ symmetry.

Now we will look at an example which is more specific in the context of $M$ theory, orientifolds and G-flux vacua [22, [17]. In M-theory one starts with $X=K 3_{1} \times K 3_{2}$

four-fold in the presence of the flux $F_{4}$. In type IIB this is an orientifold $K 3 \times \frac{T^{2}}{Z_{2}}$. This $K 3$ is $K 3_{1}$ in M-theory. The second $K 3$ which is called $K 3_{2}$ is elliptically fibered. Thus we have an M-theory on a Calabi-Yau four-fold $\mathbf{R}^{3} \times X$. We will take an Euclidean signature both in the target as well as on M5. In this case the complex divisor $D$ on which the five-brane is wrapped is a six-dimensional cycle in $X$. The 4-flux related to a 3 -form flux in type IIB theory $G_{3}=F_{3}-\phi H_{3}$ (where $F_{3}$ and $H_{3}$ are respectively RR and NS 3-forms 
and $\phi$ is a complex axion-dilaton of IIB theory) can be chosen as follows

$$
F_{4}=-\frac{1}{\phi-\bar{\phi}} G_{3} \wedge d \bar{z}_{2}+\frac{1}{\phi-\bar{\phi}} \bar{G}_{3} \wedge d z_{2},
$$

where $d z_{2}$ and $d \bar{z}_{2}$ are holomorphic and anti-holomorphic differentials along the elliptically fibered torus in $K 3_{2}$.

The examples of supersymmetric background fluxes studied in 22 and 17 require that $F_{4}$ has two legs along $K 3_{1}$ and two legs along $K 3_{2}$. This means that $H_{3}$ and $F_{3}$ have 2 legs in $K 3_{1}$ and one leg in $K 3_{2}$, in direction with coordinates $z_{1}, \bar{z}_{1}$. In our split of the 11-dimensional Euclidean space into $6+5$, the six coordinates of the M5 have to be in $D$. The 5 directions normal to the brane include $\mathbf{R}^{3}$ for the 3 directions. The remaining 2 directions, normal to $D$ are related to the $S O(2) \sim U(1)$ symmetry, which is a rotation in $z_{1}, \bar{z}_{1}$ plane. One of the examples studied in [22] and [17] is

$$
F_{4}=C \Omega \wedge d \bar{z}_{1} \wedge d \bar{z}_{2}+\bar{C} \bar{\Omega} \wedge d z_{1} \wedge d z_{2}
$$

where $C$ is a complex constant and where $\Omega(\bar{\Omega})$ is a holomorphic (anti-holomorphic) two-form on $K 3_{1}$. The flux has an $F_{z_{1}}$ as well as an $F_{\bar{z}_{1}}$ component which under the $U(1)$ transformation with a parameter $\varphi$ acquire the phase $e^{i \varphi} F_{z_{1}}$ and $e^{-i \varphi} F_{\bar{z}_{1}}$, respectively. If we would consider the fixed vacuum expectation value of the flux we would see that it violates the $U(1)$ symmetry. However, in the context in which the background flux transforms under $U(1)$ we have the following situation. The flux transforms under $U(1)$ if $C$ transforms as $e^{-i \varphi} C$ and $\bar{C}$ as $e^{i \varphi} \bar{C}$. This leaves us with the Dirac action on M5 in presence of the background fluxes which is invariant under $U(1)$.

\section{The Dirac equation and action on the M2 brane in the presence of $D=11$ fluxes}

For completeness, here we present the Green-Schwarz and purely worldvolume form of the Dirac operator on an M2 brane coupled to a $D=11$ supergravity gauge field flux

$F_{a b c d}$. Its derivation can be carried out in the same way as for the M5 brane. In the Green-Schwarz form the M2 brane fermionic equation [23] is

$$
\eta^{a b} E^{\underline{\beta}}\left[E_{b}^{a} \Gamma_{\underline{a}}(1-\bar{\Gamma})\right]_{\underline{\beta \alpha}}=0
$$

where $\bar{\Gamma}=\frac{1}{3 !} \epsilon^{a b c} \Gamma_{a b c}, \bar{\Gamma}^{2}=1, \Gamma_{a}=E \frac{\beta}{a} \Gamma_{\underline{a}}$ and $a=0,1,2$ are the worldvolume tangent space indices.

Using eqs. (16) -(19) we find that in the linear approximation in $\theta$ eq. (33) reduces to

$$
\Gamma^{a}\left(\nabla_{a}+T_{a} \underline{a b c d} F_{\underline{a b c d}}\right) \theta_{-}=0,
$$

where $\theta_{-}=\frac{1}{2}(1-\bar{\Gamma}) \theta$, being 'kappa-projected', has 16 independent components. This form of equation can also be extracted from the more general answer in [10] or from the M2 brane quadratic action of [6, 7].

To get the purely worldvolume form of the M2 brane Dirac operator with fluxes, one starts from the $M 2$ brane fermionic equation in the superembedding formulation [24, 4]

$$
\gamma^{a \alpha \beta} E_{\bar{a}}^{\underline{\beta}} v_{\underline{\beta}, \beta q^{\prime}}=0,
$$


where now $\gamma_{\alpha \beta}^{a}=\gamma_{\beta \alpha}^{a}$ are $d=3 M 2$ worldvolume symmetric gamma-matrices whose spinor indices $\alpha, \beta=1,2$ are raised and lowered by the antisymmetric unit matrices $\epsilon^{\alpha \beta}=\epsilon_{\alpha \beta}, q^{\prime}=1, \cdots, 8$ is the index of a spinor representation of the $S O(8)$ group of transformations of $d=8$ target space directions transversal to the $M 2$ brane and $v_{\beta, \beta q^{\prime}}$ are half of the components of the $\operatorname{Spin}(1,10)$ spinor Lorentz harmonics. Then, using eqs. (16) -(19) and expressions of Section 5.1 of [4], one can reduce eq. (35) to a form analogous to that for the M5 brane

$$
\gamma^{a \alpha \beta} \nabla_{a} \theta_{\beta q^{\prime}}+\frac{1}{96}\left[\left(\gamma^{i j k l} F_{i j k l}-6 \epsilon^{a b c} \gamma_{c} \gamma^{i j} F_{a b i j}\right) \theta\right]_{q^{\prime}}^{\alpha}=0 .
$$

The corresponding $M 2$ brane worldvolume Lagrangian is

$$
L_{f}^{M 2}=\frac{1}{2} \theta\left[\gamma^{a} \nabla_{a}+\frac{1}{96}\left(\gamma^{i j k l} F_{i j k l}-6 \epsilon^{a b c} \gamma_{c} \gamma^{i j} F_{a b i j}\right)\right] \theta .
$$

In eqs. (36) and (37) $\gamma_{q^{\prime} p^{\prime}}^{i j k l}=\gamma_{p^{\prime} q^{\prime}}^{i j k l}$ and $\gamma_{q^{\prime} p^{\prime}}^{i j}=-\gamma_{p^{\prime} q^{\prime}}^{i j}$ are antisymmetric products of $S O(8)$ gamma matrices $\tilde{\gamma}_{q^{\prime} p}^{i}$ and $\gamma_{p r^{\prime}}^{i}$ with the indices $i, j, k, l=1, \cdots, 8$ labeling the vector representation of $S O(8)$ and the index $p=1, \cdots, 8$ corresponding to the second spinor representation of $S O(8)$ different from that labeled by $q^{\prime}$ (see e.g. [24, 4] for details). Note that there is no difference between upper and lower $S O(8)$ indices since they all are raised and lowered by the unit symmetric matrices $\delta^{i j}, \delta^{p q}$ and $\delta^{p^{\prime} q^{\prime}}$. The gamma matrices $\gamma_{p r^{\prime}}^{i}=\tilde{\gamma}_{r^{\prime} p}^{i}$, such that $\gamma_{p r^{\prime}}^{i} \tilde{\gamma}_{r^{\prime} q}^{j}+\gamma_{p r^{\prime}}^{j} \tilde{\gamma}_{r^{\prime} q}^{i}=\delta^{i j} \delta_{p q}$, imply well known triality of the three inequivalent 8-dimensional fundamental representations of $S O(8)$.

It is interesting to note that in the presence of fluxes there is a kind of anomalous magnetic moment coupling of the worldvolume fermions to the field strengths of the fluxes. In the most straightforward way this anomalous magnetic moment coupling is seen in the Dirac equation for the M2 brane (36). In the last term of this equation $F_{a b i j}$ can be regarded as a 2 -form field strength on the $3 \mathrm{~d}$ worldvolume with $i, j$ being the indices of an internal local symmetry group. When $i, j=1,2$ take only the values of $S O(2)$, we see that this is nothing but the anomalous magnetic moment coupling of $3 \mathrm{~d}$ fermions to an electromagnetic field strength in a 3d field theory. A similar interpretation may also be given to flux terms in the Dirac operator on the M5 brane. For instance, the first flux term in (30) can be rewritten as $\gamma^{i j k} \tilde{\gamma}^{a}\left(2 \delta_{a}^{d}-m_{a}^{d}\right) F_{d i j k}=\tilde{\gamma}^{a} F_{a}^{i j} \gamma_{i j}$ (where $F_{a}^{i j}=\frac{1}{3 !} \varepsilon^{i j i_{1} j_{1} k_{1}}\left(2 \delta_{d}^{a}-m_{d}^{a}\right) F_{d i_{1} j_{1} k_{1}}$ and $\left.\gamma_{i j}=\frac{1}{3 !} \varepsilon_{i j i_{1} j_{1} k_{1}} \gamma^{i_{1} j_{1} k_{1}}\right)$. One can notice that the 'anomalous magnetic moment' term $F_{a}^{i j} \gamma_{i j}$ has the form similar to that of the $S O(5)$ connection $\Omega_{a}^{i j} \gamma_{i j}$ which enters the covariant derivative of the M5 brane Dirac operator (30).

\section{Conclusion}

Thus we have derived here the explicit dependence on fluxes in the fermionic action on the M5 brane in a generic background. We have shown that there are two types of fluxes which enter the Dirac action. One of them transforms as an antisymmetric rank 3 tensor and another one as a vector of the R-symmetry group $S O(5)$ of the M5-brane, and in particular of its $\mathrm{SO}(2)$ subgroup. This poses a question: what happens in general with the condition $\chi_{D}=1$ derived in [13] and studied more recently in [16] and [17. In examples of compactification shown in [17] the answer was that $\chi_{D} \geq 1$ might provide the nonvanishing superpotential. It is not yet known how to generalize Witten's analysis for the 
Dirac operator in eq. (28). This equation is a generalization of a simple Dirac equation used in [13] under the condition that there is no background flux, $F_{4}=0$ and there is no chiral 2 -form on M5, $b_{2}=0$. When the background fluxes and world-volume 2 -forms are present, in general, the analysis of the instanton corrections has to be redone. The immediate reason for this is the fact that background fluxes are necessarily required for stabilization of the dilaton-axion and complex structure moduli [25].

The importance of this topic has to do with the fact that the only known way at present in which string theory and higher-dimensional supergravities may, possibly, address the current cosmological observations, require stabilization of moduli. The most difficult part, stabilization of Kähler moduli, is based on non-perturbative instanton corrections to the superpotential discussed here. Clarification of the restrictions on compactification manifolds which might provide such non-perturbative superpotentials might lead to a significant progress in string cosmology.

\section{Acknowledgements}

We are grateful to F. Denef and B. Florea who suggested to look for the Dirac operator with fluxes on M5 in the context of instanton corrections, and to E. Bergshoeff, I. Bandos, J. Gomis, S. Gukov, S. Kachru, G. Moore, A. Kashani-Poor, and S. Sethi, A. Tomasiello, S. Trivedi and A. Van Proeyen for clarifying discussions. The work of R. K. was supported by NSF grant 0244728. The work of D. S. was supported by the EU MRTN-CT-2004005104 grant 'Forces Universe', and by the MIUR contract no. 2003023852.

\section{Appendix}

In our notation the underlined indices correspond to $D=11$ target superspace and not underlined ones correspond to the M5 brane worldvolume. The indices from the beginning of the Latin and Greek alphabet are vector and spinor tangent (super)space indices, while the indices from the middle of the Latin and Greek alphabet are that of local curved coordinates. The letters $i, j, k$ and $p, q, r, s$ stand, respectively for vector and spinor $\operatorname{Spin}(5)$ indices.

We use the form of the $D=11 \Gamma$-matrices and of the charge conjugation matrices $C_{\underline{\alpha} \underline{\beta}}=C \underline{\alpha} \underline{\beta}$ which reflects the embedding of the M5 brane $6 d$ worldvolume into $N=1$, $D \equiv 11$ superspace

$$
\begin{gathered}
\Gamma_{\underline{\alpha} \underline{\beta}}^{a}=\left(\begin{array}{cc}
\gamma_{\alpha \beta}^{a} C_{p q} & 0 \\
0 & \tilde{\gamma}^{a \alpha \beta} C^{p q}
\end{array}\right), \quad a=0,1, \ldots, 5, \quad \alpha, \beta=1,2,3,4, \\
\Gamma_{\underline{\alpha} \underline{\beta}}^{i}=\left(\begin{array}{cc}
0 & \delta_{\beta}^{\alpha}\left(\gamma^{i}\right)_{q}^{p} \\
-\delta_{\alpha}^{\beta}\left(\gamma^{i}\right)_{p}^{q} & 0
\end{array}\right), \quad i=1, \ldots, 5, \quad q, p=1,2,3,4, \\
C_{\underline{\alpha} \underline{\beta}}=C^{\underline{\alpha} \underline{\beta}}=\left(\begin{array}{cc}
0 & \delta_{\beta}^{\alpha} \delta_{q}^{p} \\
-\delta_{\alpha}^{\beta} \delta_{p}^{q} & 0
\end{array}\right) .
\end{gathered}
$$

In (39) $\left(\gamma^{i}\right)_{p}^{q}=C^{q s}\left(\gamma^{i}\right){ }_{s}^{r} C_{r p}$ are $U S p(4) \sim S O(5)$ gamma-matrices and $C^{q s}=C_{q s}$ are charge conjugation matrices. The matrices $\left(\gamma^{i}\right)_{q p}=\left(\gamma^{i}\right)_{q}^{r} C_{r p}$ and $C^{q s}$ are antisymmetric.

$\tilde{\gamma}_{b}^{\alpha \beta}$ and $\gamma_{\alpha \beta}^{a}$ are antisymmetric $d=6 \operatorname{Spin}(1,5) \gamma$-matrices having the following properties

$$
\gamma_{\alpha \gamma}^{a} \tilde{\gamma}^{b \gamma \beta}+\gamma_{\alpha \gamma}^{b} \tilde{\gamma}^{a \gamma \beta}=2 \delta_{\alpha}^{\beta} \eta^{a b}, \quad \operatorname{tr}\left(\gamma^{a} \tilde{\gamma}^{b}\right)=4 \eta^{a b}, \quad \gamma_{a \alpha \beta} \gamma_{\gamma \delta}^{a}=-2 \epsilon_{\alpha \beta \gamma \delta},
$$




$$
\begin{aligned}
& \gamma_{\alpha \beta}^{a b c}=\gamma_{\beta \alpha}^{a b c} \equiv\left(\gamma^{[a} \tilde{\gamma}^{b} \gamma^{c]}\right)_{\alpha \beta}=-\frac{1}{6} \epsilon^{a b c d e f}\left(\gamma_{d e f}\right)_{\alpha \beta}, \\
& \tilde{\gamma}^{a b c \alpha \beta}=\tilde{\gamma}^{a b c \beta \alpha} \equiv\left(\tilde{\gamma}^{[a} \gamma^{b} \tilde{\gamma}^{c]}\right)^{\alpha \beta}=\frac{1}{6} \epsilon^{a b c d e f} \tilde{\gamma}_{d e f}^{\alpha \beta},
\end{aligned}
$$

The Lorentz spinor harmonics (24)

$$
v_{\underline{\underline{\alpha}}}^{\underline{\beta}}=\left(v_{\underline{\alpha}}^{\alpha p}, v_{\underline{\alpha}, \beta q}\right), \quad C \underline{\underline{\alpha}} \underline{\underline{\gamma}} v_{\underline{\alpha}}^{\underline{\beta}} v_{\underline{\gamma}}^{\underline{\delta}}=C \underline{\beta} \underline{\delta}, \quad v^{\underline{\alpha}} \underline{\underline{\gamma}}=C{ }^{\alpha \alpha^{\prime}} v_{\underline{\alpha}^{\prime}} \underline{\gamma}^{\prime} C_{{\underline{\gamma^{\prime}}}^{\gamma}}
$$

are auxiliary worldvolume fields. They are related to the pullback $E \frac{A}{a}(Z(\xi))$ of the $D=11$ supervielbein (5) by the following equations

$$
E_{a} \underline{\alpha}_{\underline{\alpha}}^{\alpha p}=0, \quad \Gamma_{\underline{\gamma} \underline{\delta}}^{a} E_{a} \underline{a}+\Gamma_{\underline{\gamma} \underline{\delta}}^{i} u_{i}^{\underline{a}} \equiv \Gamma_{\underline{\gamma} \underline{\delta}}^{\underline{b}} u_{\underline{b}}^{\underline{a}}=v_{\underline{\underline{\gamma}}}^{\underline{\alpha}} \Gamma_{\underline{\alpha} \underline{\beta}}^{\underline{a}} v_{\underline{\delta}}^{\underline{\beta}},
$$

where $u_{i}{ }^{\underline{a}}(\xi)$ are a set of five $D=11$ Lorentz vectors with indices $(i=1, \cdots, 5)$ belonging to the vector representation of $\operatorname{Spin}(5) . u_{i}{ }^{\underline{a}}(\xi)$ are defined to be orthogonal to $E_{a} \underline{a}$, i.e.

$$
E_{a} \underline{a} u_{i}^{\underline{b}} \eta_{\underline{a b}}=0
$$

and complement the latter to an $S O(1,10)$ matrix (also called Lorentz vector harmonics)

$$
u_{\underline{b}}^{\underline{a}}=\left(E_{a} \underline{a}, u_{i}^{\underline{a}}\right), \quad u_{\underline{b}}^{\underline{a}} u_{\underline{d}}^{\underline{c}} \eta_{\underline{a c}}=\eta_{\underline{b d}}, \quad u_{\underline{b}}^{\underline{a}} u_{\underline{d}}^{\underline{c}} \eta^{\underline{b d}}=\eta \underline{\underline{a c}} .
$$

Using the relations (38)-(45) one can show that the fermionic equations (31) and (23) are equivalent [2, 4, with the projector matrix $\frac{1}{2}(1-\bar{\Gamma})$ (9) having the following form in terms of the Lorentz spinor harmonics (24) and the self-dual tensor field $h_{a b c}$

$$
\frac{1}{2}(1-\bar{\Gamma})_{\underline{\alpha} \underline{\beta}}=v_{\underline{\alpha}}^{\beta p}\left(v_{\underline{\beta}, \beta p}+C_{p q} h_{a b c} \gamma_{\beta \gamma}^{a b c} v_{\underline{\beta}}^{\gamma q}\right) .
$$

\section{References}

[1] I. A. Bandos, K. Lechner, A. Nurmagambetov, P. Pasti, D. P. Sorokin and M. Tonin, "Covariant action for the super-five-brane of M-theory," Phys. Rev. Lett. 78, 4332 (1997) arXiv:hep-th/9701149.

M. Aganagic, J. Park, C. Popescu and J. H. Schwarz, Nucl. Phys. B496 (1997) 191. arXiv:hep-th/9701166.

[2] P. S. Howe and E. Sezgin, " $\mathrm{D}=11, \mathrm{P}=$ 5", Phys. Lett. B 394 (1997) 62 arXiv:hep-th/9611008 .

P. S. Howe, E. Sezgin and P. C. West, "Covariant field equations of the M-theory five-brane," Phys. Lett. B 399, 49 (1997) arXiv:hep-th/9702008.

[3] I. A. Bandos, K. Lechner, A. Nurmagambetov, P. Pasti, D. P. Sorokin and M. Tonin, "On the equivalence of different formulations of the M theory five-brane," Phys. Lett. B 408, 135 (1997) arXiv:hep-th/9703127.

[4] D. P. Sorokin, "Superbranes and superembeddings," Phys. Rept. 329, 1 (2000) arXiv:hep-th/9906142. 
[5] M. Grana, "D3-brane action in a supergravity background: The fermionic story," Phys. Rev. D 66, 045014 (2002) arXiv:hep-th/0202118.

[6] D. Marolf, L. Martucci and P. J. Silva, "Actions and fermionic symmetries for Dbranes in bosonic backgrounds," JHEP 0307, 019 (2003) arXiv:hep-th/0306066.

[7] D. Marolf, L. Martucci and P. J. Silva, "Fermions, T-duality and effective actions for D-branes in bosonic backgrounds," JHEP 0304, 051 (2003) arXiv:hep-th/0303209.

[8] M. T. Grisaru and M. E. Knutt, "Norcor vs the abominable gauge completion," Phys. Lett. B 500, 188 (2001) arXiv:hep-th/0011173.

[9] P. Claus, R. Kallosh and A. Van Proeyen, "M 5-brane and superconformal $(0,2)$ tensor multiplet in 6 dimensions," Nucl. Phys. B 518, 117 (1998) arXiv:hep-th/9711161.

[10] B. de Wit, K. Peeters and J. Plefka, "Superspace geometry for supermembrane backgrounds," Nucl. Phys. B 532, 99 (1998) arXiv:hep-th/9803209.

[11] N. Drukker, D. J. Gross and A. A. Tseytlin, "Green-Schwarz string in AdS(5) x S(5): Semiclassical partition function," JHEP 0004, 021 (2000) arXiv:hep-th/0001204.

[12] J. Gomis, K. Kamimura and P. K. Townsend, "Non-relativistic superbranes," JHEP 0411, 051 (2004) arXiv:hep-th/0409219.

[13] E. Witten, "Non-Perturbative Superpotentials In String Theory," Nucl. Phys. B 474, 343 (1996) arXiv:hep-th/9604030.

[14] S. Kachru, R. Kallosh, A. Linde and S. P. Trivedi, "De Sitter vacua in string theory," Phys. Rev. D 68, 046005 (2003) arXiv:hep-th/0301240.

[15] F. Denef, M. R. Douglas and B. Florea, "Building a better racetrack," JHEP 0406 , 034 (2004) arXiv:hep-th/0404257.

[16] D. Robbins and S. Sethi, "A barren landscape," arXiv:hep-th/0405011.

[17] L. Gorlich, S. Kachru, P. K. Tripathy and S. P. Trivedi, "Gaugino condensation and nonperturbative superpotentials in flux compactifications," arXiv:hep-th/0407130.

[18] B. S. DeWitt, "Quantum Theory Of Gravity. Ii. The Manifestly Covariant Theory," Phys. Rev. 162, 1195 (1967); R. E. Kallosh, "The Renormalization In Nonabelian Gauge Theories," Nucl. Phys. B 78, 293 (1974); M. T. Grisaru, P. van Nieuwenhuizen and C. C. Wu, "Background Field Method Versus Normal Field Theory In Explicit Examples: One Loop Divergences In S Matrix And Green's Functions For Yang-Mills And Gravitational Fields," Phys. Rev. D 12, 3203 (1975).

[19] E. Cremmer and S. Ferrara, "Formulation Of Eleven-Dimensional Supergravity In Superspace," Phys. Lett. B 91, 61 (1980).

[20] D. Tsimpis, "Curved 11D supergeometry," JHEP 0411, 087 (2004) arXiv:hep-th/0407244. 
[21] I. A. Bandos, J. A. de Azcarraga, J. M. Izquierdo and J. Lukierski, "D = 4 supergravity dynamically coupled to a massless superparticle in a superfield Lagrangian approach," Phys. Rev. D 67, 065003 (2003) arXiv:hep-th/0207139.

[22] K. Dasgupta, G. Rajesh and S. Sethi, "M theory, orientifolds and G-flux," JHEP 9908, 023 (1999) arXiv:hep-th/9908088.

[23] E. Bergshoeff, E. Sezgin and P. K. Townsend, "Supermembranes And ElevenDimensional Supergravity," Phys. Lett. B 189, 75 (1987).

E. Bergshoeff, E. Sezgin and P. K. Townsend, "Properties Of The ElevenDimensional Super Membrane Theory," Annals Phys. 185, 330 (1988).

[24] I. Bandos, P. Pasti, D. Sorokin, M. Tonin and D. Volkov, "Superstrings and supermembranes in the doubly supersymmetric geometrical approach," Nucl. Phys. B446, 79 (1995) arxiv:hep-th/9501113.

[25] S. B. Giddings, S. Kachru and J. Polchinski, "Hierarchies from fluxes in string compactifications," Phys. Rev. D66, 106006 (2002) arXiv:hep-th/0105097. 